\title{
ENERGÍAS RENOVABLES ALTERNATIVAS, UN RETO PARA EL PERÚ
}

\author{
Pedro Gamio Aita*
}

\section{REGULACIÓN ELÉCTRICA}

La actividad propiamente eléctrica se inicia en 1884, con la operación de la primera central hidroeléctrica en la provincia de Huaraz, distrito de Yangas, construida por una empresa minera. Es en el gobierno del general Miguel Iglesias, en 1886, con la empresa privada Peruvian Electric Construction and Supply (PECS), que entra en operación la primera central térmica que permitió el alumbrado público en la Plaza de Armas y algunas calles del centro de Lima. Después de 170 años todavía en el país no logramos pleno acceso a la energía. La matriz se basa principalmente en hidroelectricidad y gas natural, con una pequeña participación de solo $5 \%$ de energías renovables alternativas.

El sector eléctrico peruano actual tiene como base la Ley de Concesiones Eléctricas (en adelante LCE), aprobada mediante Decreto Ley N. ${ }^{\circ} 25844$, vigente desde el 1992, y su Reglamento, aprobado mediante Decreto Supremo N. ${ }^{009-1993-E M, ~ a s i ́ ~ c o m o ~ s u s ~ m o d i f i c a t o r i a s ~ y ~ n o r m a s ~ c o m-~}$

\footnotetext{
* Abogado, Magíster en Gestión de Políticas Públicas por la Universidad Carlos III de España y egresado de la Maestría de Ciencia Política de la Pontificia Universidad Católica del Perú (PUCP). Ha realizado estudios de posgrado en Gestión Empresarial en Energía en ESAN. Tiene estudios de especialización en Legislación ambiental, por la Universidad Politécnica de Madrid, España, y en Contratos de Energía por el Petroleum Institute of Texas. Asimismo, ha realizado curso de especialización en Gestión de la Innovación, Harvard University.

Actualmente, es Presidente del Panel Asesor de Monitoreo Independiente del Proyecto Camisea, por encargo del Eximbank. Es consultor de GIZ, agencia alemana de cooperación internacional. Fue consultor de SNV, Servicio Holandés de Cooperación al Desarrollo. Asimismo, es integrante del Consejo Directivo de la Plataforma Latinoamericana de Energías Renovables (PLESE). Ha sido parte del equipo negociador del Perú en la COP 20 y coordinador de Energía, así como participante en la COP 21 y COP 23.

Se desempeńó como viceministro de Energía del Perú entre los años 2006 y 2008, como consultor de la Fundación Konrad Adenauer, del Banco Mundial, del BID, PNUD, WWF, OEFA y CEPLAN en materia de energía y medioambiente. Fue director para América Latina de Global Village Energy Partnership y de INDUFOR con el Gobierno de Finlandia y el IICA; Chief Technical Advisor o asesor principal de dos fondos concursables, que han permitido la ejecución de 50 proyectos innovadores y replicables con tecnologías renovables en zonas rurales y de extrema pobreza en Latinoamérica.

Correo electrónico: pedrogamioa@gmail.com
} 
plementarias. Esta Ley marcó la liberalización y privatización del mercado eléctrico peruano desde 1992, estableciendo la división de las actividades del sector eléctrico en generación, transmisión, distribución y comercialización, para las cuales se podían otorgar concesiones y autorizaciones, actuando el Estado como ente regulador (Organismo Supervisor de la Inversión en Energía y Minería [OSINERGMIN], 2016). A partir de esta Ley, nacieron otras que determinaron la estructura del mercado eléctrico actual.

La reforma legal del sector eléctrico se concretó junto a la privatización de la mayor parte de los activos que el Estado mantenía en el mercado eléctrico. Eso trajo inversiones y la mejora de la calidad del servicio público en Lima, la capital del país. El capital privado involucró mayor innovación y eficiencia en las prestaciones. Mediante la inversión privada en la actividad eléctrica, se mejoró la infraestructura, se inicia la construcción de nuevas centrales y más líneas de transmisión. A continuación, se realizará una breve descripción de las normativas principales, de prospectiva, planes y normativas relacionadas con la electricidad y los recursos energéticos renovables.

\section{Ley de Concesiones Eléctricas (Decreto Ley N.'25844)}

Mediante la LCE se estableció que se constituyeran servicios públicos de electricidad: a) El suministro regular de energía eléctrica para uso colectivo o destinado al uso colectivo, hasta los límites de potencia fijados por el Reglamento; $y, b)$ La transmisión y distribución de electricidad. Además, se instituyó que el Servicio Público de Electricidad es de utilidad pública (Decreto Ley N. ${ }^{\circ}$ 25844). Por lo tanto, se re- quiere concesión definitiva para cada una de las siguientes actividades:

- La generación de energía eléctrica que utilice recursos hidráulicos, con potencia instalada mayor de $500 \mathrm{~kW}$.

- La transmisión de energía eléctrica, cuando las instalaciones afecten bienes del Estado y/o requieran la imposición de servidumbre por parte de este.

- La distribución de energía eléctrica con carácter de servicio público de electricidad, cuando la demanda supere los $500 \mathrm{~kW}$.

- La generación de energía eléctrica con recursos energéticos renovables conforme a la Ley de la materia, con potencia instalada mayor de $500 \mathrm{~kW}$ (Decreto Ley N. ${ }^{\circ} 25844$ ).

Así, a través de este dispositivo legal, se estableció que las concesiones y autorizaciones sean otorgadas por el Ministerio de Energía y Minas, que instaura para tal efecto un Registro Único de Concesiones Eléctricas a nivel nacional, en el cual se inscriben las concesiones otorgadas y las solicitudes en trámite presentadas ante el Ministerio y los gobiernos regionales, y que las actividades de generación, transmisión y distribución que no requieren de concesión ni autorización pueden ser efectuadas libremente cumpliendo las normas técnicas y disposiciones de conservación del medio ambiente y del patrimonio cultural de la nación (Decreto Ley N. ${ }^{\circ}$ 25844).

\section{Ley Antimonopolio y Antioligopolio del Sector Eléctrico ([LAAOSE], Ley N.²6876)}

Esta Ley establece que, para evitar los actos de concentración que tengan por efecto disminuir, dañar o impedir la competencia y la libre concurrencia en los mercados de las acti- 
vidades de generación y/o de transmisión y/o de distribución de energía eléctrica o en los mercados relacionados, las concentraciones de tipo vertical u horizontal que se produzcan se sujetarán a un procedimiento de autorización previa (Ley N. 26876).

Se entiende por concentración, la realización de los siguientes actos: la fusión; la constitución de una empresa en común; la adquisición directa o indirecta del control sobre otras empresas, a través de la adquisición de acciones, participaciones o a través de cualquier otro contrato o figura jurídica que confiera el control directo o indirecto de una empresa. incluyendo la celebración de contratos de asociación joint venture —asociación en participación-, uso o usufructo de acciones y/o participaciones, contratos de gerencia, de gestión, y de sindicación de acciones o cualquier otro contrato de colaboración empresarial similar, análogo y/o parecido y de consecuencias similares.

Asimismo, la adquisición de activos productivos de cualquier empresa que desarrolle actividades en el sector o cualquier otro acto, contrato o figura jurídica incluyendo legados, por virtud del cual se concentren sociedades, asociaciones, acciones, partes sociales, fideicomisos o activos en general, que se realice entre competidores, proveedores, clientes, accionistas o cualesquiera otros agentes económicos (Ley N. ${ }^{\circ}$ 26876).

\section{Ley para Asegurar el Desarrollo Eficiente de la Generación Eléctrica (Ley N..2 28832)}

Esta Ley tuvo como objetivo asegurar el desarrollo eficiente de la generación eléctrica, perfeccionando las reglas establecidas en la Ley de Concesiones Eléctricas con la finalidad de:
- Asegurar la suficiencia de generación eficiente que reduzca la exposición del sistema eléctrico peruano a la volatilidad de precios y a los riesgos de racionamiento prolongado por falta de energía; asegurando al consumidor final una tarifa eléctrica más competitiva.

- Reducir la intervención administrativa para la determinación de los precios de generación mediante soluciones de mercado.

- Adoptar las medidas necesarias para propiciar la efectiva competencia en el mercado de generación.

- Introducir un mecanismo de compensación entre el SEIN y los sistemas aislados para que los precios en barra de estos últimos incorporen los beneficios del gas natural y reduzcan su exposición a la volatilidad del mercado de combustibles (Ley N. ${ }^{\circ}$ 28832).

La norma marca las pautas respecto de los contratos entre el generador y el distribuidor, destinadas al servicio público de electricidad, mencionando que, las ventas de electricidad de generador a distribuidor pueden ser: a) mediante contratos sin licitación, donde los precios no podrán ser superiores a los precios en barra, desarrollado en la Ley de Concesiones Eléctricas; y, b) mediante contratos resultantes de licitaciones.

Asimismo, se desarrolló la figura de la licitación como medida preventiva para el abastecimiento oportuno de energía eléctrica, desarrollando los plazos, las bases, el precio, las condiciones y obligaciones de los contratos derivados de un proceso de licitación (Ley N. ${ }^{\circ}$ 28832).

Respecto del mercado de corto plazo, la norma menciona que la compra y venta de energía en 
el mercado de corto plazo se efectúa en función a los costos marginales de corto plazo nodales. Se menciona que los generadores, distribuidores y grandes usuarios libres, en caso de que fuera necesario, deberán constituir fideicomisos u otras garantías de realización inmediata como respaldo de los retiros de capacidad y energía que efectúen del mercado de corto plazo, de tal manera que se garantice el pago oportuno en dicho mercado (Ley N. ${ }^{\circ} 28832$ ).

Un aspecto importante de la Ley en mención es que desarrolla la naturaleza del Comité de Operación Económica del Sistema (COES) en los siguientes términos:

El COES tiene por finalidad coordinar la operación de corto, mediano y largo plazo del SEIN al mínimo costo, preservando la seguridad del sistema, el mejor aprovechamiento de los recursos energéticos, así como planificar el desarrollo de la transmisión del SEIN y administrar el Mercado de Corto Plazo. El COES es una entidad privada, sin fines de lucro y con personería de Derecho Público. Está conformado por todos los Agentes del SEIN y sus decisiones son de cumplimiento obligatorio por los Agentes (Ley N. ․ 28832).

Es importante recalcar que esta Ley trajo consigo la definición de generación distribuida e impulsó las medidas para la promoción de la generación distribuida y cogeneración eficientes, y menciona lo siguiente (Ley N. ${ }^{\circ} 28832$ ):

- La venta de sus excedentes no contratados de energía al mercado de corto plazo, asignados a los generadores de mayor transferencia (de compra o negativa) en dicho mercado; $y$,

- El uso de las redes de distribución pagando únicamente el costo incremental incurrido.
Política Energética Nacional del Perú, 20102040 (Decreto Supremo N. . 064- 2010-EM)

Con fecha 24 de noviembre de 2010 se aprobó la Política Energética Nacional del Perú para el periodo 2010-2040, tomando como referencia los lineamientos del Plan Estratégico de Desarrollo Nacional - Plan Perú 2021, elaborado por el Centro de Planeamiento Estratégico (Ceplan). En esta política se promueve la eficiencia energética y el desarrollo de las energías renovables a nivel local, regional y nacional (Decreto Supremo N. . 064-2010-EM).

Los objetivos de la política son los siguientes:

- Contar con una matriz energética diversificada, con énfasis en las fuentes renovables y la eficiencia energética.

- Contar con un abastecimiento energético competitivo.

- Acceso universal al suministro energético.

- Contar con la mayor eficiencia en la cadena productiva y de uso de la energía.

- Lograr la autosuficiencia en la producción de energéticos.

- Desarrollar un sector energético con mínimo impacto ambiental y bajas emisiones de carbono en un marco de desarrollo sostenible.

- Desarrollar la industria del gas natural, y su uso en actividades domiciliarias, transporte, comercio e industria, así como la generación eléctrica eficiente.

- Fortalecer la institucionalidad del sector energético.

- Integrarse con los mercados energéticos de la región, que permita el logro de la visión de largo plazo (Decreto Supremo N. ${ }^{\circ} 064-$ 2010-EM). 
En su exposición de motivos se menciona que existe una relación muy estrecha entre crecimiento económico y consumo de energía y que la energía, presente en todas las actividades productivas, constituye un indicador del nivel de desarrollo del país (Gobierno del Perú, 2012).

Se menciona, además, que la energía también es responsable de la emisión de gases de efecto invernadero (GEI), que es una de las causas del cambio climático, el cual es de interés a nivel nacional e internacional. Se concluye que la política es necesaria toda vez que se debe afrontar las necesidades futuras de energía y posibilitar el desarrollo sostenible del país (Gobierno del Perú, 2012).

\section{Plan Energético Nacional 2014-2025}

El Plan Energético Nacional 2014-2025, documento elaborado por el Ministerio de Energía y Minas (MINEM) con el apoyo de una comisión consultiva, tuvo como finalidad plantear una propuesta de plan para el mediano plazo (hasta el horizonte 2025) y la preparación de una propuesta para institucionalizar el proceso de planeamiento energético en el Perú (Ministerio de Energía y Minas [MINEM], 2014).

En ese sentido, se proyecta el consumo final de energía, la oferta de energía, la infraestructura de transporte y distribución, las medidas de inclusión social energética, el empleo de energías renovables, la eficiencia energética, la integración energética, el cambio climático y el panorama de las inversiones. Sin embargo, no se establecen objetivos claros ni medidas concretas para alcanzarlos a largo plazo (MINEM, 2014).
Decreto Legislativo N. ${ }^{\circ}$ 1002: Generación de Electricidad con Recursos Energéticos Renovables Alternativos (RER)

El Decreto Legislativo N. ${ }^{\circ} 1002$ declaró de interés nacional y de necesidad pública el desarrollo de nueva generación eléctrica mediante el uso de recursos energéticos renovables, tales como energía eólica, solar, bioenergía, mareomotriz y geotermia e introdujo incentivos para promover la inversión en generación eléctrica a partir de estos recursos.

Se estableció un costo marginal de cero para las centrales RER, por lo tanto tendrían prioridad en el despacho. Además, se ha establecido un esquema de devolución de impuestos en los proyectos de RER, en el cual se realiza un mecanismo de depreciación acelerada de hasta el $20 \%$ de los gastos de inversión en maquinaria, equipos y obras de construcción civil que influye en la determinación de la base imponible para efectos tributarios. El régimen de depreciación acelerada regulado por el Decreto Legislativo N. ${ }^{\circ} 1058$ es un beneficio tributario que es de aplicación únicamente respecto de las maquinarias, equipos y obras civiles adquiridos y/o construidos para la instalación y operación de centrales de generación de energía eléctrica a base de recursos hídricos u otros recursos renovables.

En ese sentido, el régimen de depreciación acelerada, regulado por el Decreto Legislativo N.o 1058, no es de aplicación a todos los sujetos que se dediquen a la actividad de generación eléctrica con recursos hídricos u otros recursos renovables, sino sólo a aquellos que inviertan en la construcción de nuevas centrales de generación eléctrica que entren en operación comercial a partir de la entrada en vigencia de dicho Decreto Legislativo y únicamente respecto de las ma- 
quinarias, equipos y obras civiles adquiridos y/o construidos a partir de la misma fecha para la instalación y operación de tales centrales de generación eléctrica.

Cabe tener en cuenta también que no sería un propósito de la norma beneficiar con el otorgamiento del beneficio tributario en cuestión a aquellos que se dedican a la actividad de generación eléctrica que compran una central de generación eléctrica a base de recursos hídricos $\mathrm{u}$ otros recursos renovables que ya se encuentren en operación.

En cuanto a los impuestos al consumo, un beneficio relevante para el sector está dado por la posibilidad de acceder al régimen de recuperación anticipada del impuesto general a las ventas (IGV), bajo el cual el Estado restituye a los inversionistas el IGV de las compras locales e importaciones efectuadas durante la etapa preoperativa, es decir antes del inicio de la explotación comercial del proyecto. A tal efecto, el proyecto de generación debe tener un periodo preoperativo de, por lo menos, dos años, y la empresa debe comprometerse a invertir al menos cinco millones de dólares en bienes de capital, servicios, contratos de construcción e importaciones asociadas al proyecto, pues dota de liquidez al negocio, al acceder el inversionista a la restitución del IGV de sus compras sin tener que esperar a que el proyecto empiece a generar ingresos afectos con este tributo.

Adicionalmente, este DL abrió puertas a las subastas RER organizadas por el MINEM, las cuales permitieron incrementar la participación de tecnologías renovables en la matriz energética peruana a un precio fijo, garantizado por el Estado. De ese modo, los titulares de las instalaciones que resultaron adjudicados en las subastas por tecnología RER debían vender, total o parcialmente, la producción de energía eléctrica en el mercado de corto plazo, al precio que resulte en dicho mercado, complementado con la prima fijada por el Organismo Supervisor de la Inversión en Energía y Minería (Osinergmin), en caso de que el costo marginal resultara menor que la tarifa determinada por el ente regulador (Decreto Legislativo N.o 1002).

Los ingresos de los generadores RER se encuentran garantizados por los ingresos marginales (venta de energía a costos marginales del COES) que son complementados por los ingresos por prima (sobrecargos en la tarifa al usuario final), donde los ingresos garantizados han sido cubiertos mes a mes por los ingresos del mercado y por los ingresos que pagan los usuarios. Según entrevista a Riquel Mitma ${ }^{1}$, si bien desde el año 2008, mediante el Decreto Legislativo N. ${ }^{o}$ 1002, marco regulatorio especial para promocionar las energías renovables no convencionales, el país ha obtenido resultados muy importantes porque las subastas de energías renovables han permitido lograr visibilidad del Perú a nivel mundial en cuanto a la promoción de las energías renovables.

Al no existir un plan de desarrollo de las energías renovables, no obstante, el mandato legal del Decreto Legislativo N. ${ }^{\circ} 1002$, no es posible mitigar la incertidumbre existente sobre la continuidad en el desarrollo de las diferentes tecnologías renovables adjudicadas en las subastas. El mismo Decreto Legislativo N.o 1002 establece expresamente la obligación de aprobar un plan nacional de energías renovables, a la fecha esto no se ha cumplido. Sí se

1 Profesor de Postgrado de la Universidad de ESAN. Ingeniero Electricista de la Universidad Nacional de Ingeniería (Perú); Magíster en Finanzas de la Universidad del Pacífico (Perú); Máster en Economía y Regulación de los Servicios Públicos de la Universidad de Barcelona (España). 
han desarrollado cuatro subastas, con un resultado exitoso, tanto por el nivel de participación como por la reducción de los costos. Este proceso ha quedado congelado en el tiempo, el Ministerio no ha vuelto a hacer convocatorias, perdiéndose así la oportunidad de avanzar con la mayor participación de las energías renovables no convencionales.

En el Perú se dio un gran avance con la aprobación del Decreto Legislativo N.o 1002, en mayo de 2008, el marco promotor de las energías renovables no convencionales más completo de la región. A decir de la Agencia Internacional de Energía Renovable (IRENA, por sus siglas en inglés), «En América Latina se han identificado diseños de políticas innovadoras, en particular los que combinan las subastas con otros mecanismos de regulación» (2015, p.). Sin embargo, estamos avanzando muy lentamente; otros países de la región nos han superado, sin tener necesariamente un marco tan promotor. Según sostiene la Agencia Internacional de Energía (AIE), en el 2050 la energía más importante será la energía solar. Se necesita decisión política y mejor gerencia del país, visión de lo que cuesta o costará no tomar determinadas decisiones. El Perú puede lograr una matriz desconcentrada, más diversa y competitiva, y con menor huella de carbono. Hagamos el cambio, busquemos la resiliencia. Las siguientes generaciones lo esperan de nosotros.

A lo anterior cabe añadir que en el Marco del Acuerdo de París, suscrito en el 2015 y luego ratificado por el Perú en el 2016, el país se comprometió a realizar toda acción necesaria para reducir la emisión de gases de efecto invernadero (GEI), para así coadyuvar al compromiso mundial de los 195 países firmantes frente al cambio climático: de hacer todo lo posible para que la temperatura global no suba por encima de $1,5^{\circ} \mathrm{C}$. Sin embargo, a pesar de que existen las disposiciones internas y los acuerdos internacionales y que los recursos energéticos renovables (RER) constituyen una fuente de generación eléctrica limpia, es decir, sin emisiones de GEI, y que el Perú cuenta con importantes recursos como la energía solar, eólica, biomasa, geotérmica, hidráulica, y mareomotriz, aún no se logra incorporarlas al Sistema Eléctrico Interconectado Nacional (SEIN) como fuentes generación con importantes aportes, debido a que existen restricciones y barreras que impiden la participación activa de los RER en la generación eléctrica.

\section{BARRERAS A LAS RENOVABLES ALTERNATIVAS}

Específicamente en el caso del sector eléctrico, se pueden mencionar limitantes para el desarrollo de proyectos de energías renovables no convencionales. El primer gran problema en el sector eléctrico es la falta de una planificación estratégica con metas claras y el segundo problema es la fuerte presencia de lobbies. Hasta el momento no se ha logrado concretar una política energética a largo plazo en la que participen los diferentes actores y se establezcan objetivos y responsabilidades para alcanzarlos. En ese sentido, no se define una meta específica para la inserción de las energías renovables dentro de la matriz eléctrica que sea sustentable técnica, económica y ambientalmente y además consensuada por los diferentes actores. A esto se suman los tradicionales lobbies, que resisten el cambio.

Si bien, los precios de las tecnologías renovables, como la tecnología solar fotovoltaica y eólica, han disminuido enormemente a nivel mundial, los precios del mercado spot del 
sector eléctrico peruano no son competitivos, en el 2008 se contaba con un precio de 88.21 US\$/MW.h, siendo que el precio del mercado spot ha disminuido paulatinamente hasta 8.91 US\$/MW.h en el 2019.

Esta distorsión en el mercado spot se origina en la ausencia de una declaración de costos reales por parte de las generadoras térmicas a gas natural. Esto provoca distorsiones. Los 7 millones y medio de clientes regulados terminan pagando en el recibo de luz el doble por el rubro generación, a diferencia de 2500 clientes libres. Esta situación ha comenzado a ser corregida recientemente por el Osinergmin para desarrollar una libre competencia en el mercado eléctrico. Además, se ha relanzado el mercado secundario, que busca flexibilizar la obligación de mantener una capacidad de transporte contratada en el gasoducto. Esto es relevante para que las generadoras térmicas puedan obtener ingresos por la capacidad contratada que no usan.

De otro lado, hay barreras como la definición reglamentaria de potencia en el Perú y la falta de regulación del despacho por bloque horario que sigue cerrando el camino a la energía solar para poder atender al mercado libre o de grandes consumidores de energía. Sólo pueden atender al sistema regulado, por medio de licitaciones o subastas.

En el caso específico de tecnologías renovables, como la eólica y solar fotovoltaica, no obstante, la disminución considerable de sus precios a nivel mundial, desde el 2013 no se han efectuado nuevas subastas en el país y se espera que estas tecnologías ingresen al mercado de los clientes libres, hoy sólo tienen presencia en el mercado regulado.

Por otro lado, tecnologías que tienen altos costos de inversión y que aún no se han desarrolla- do en el Perú, como las centrales geotérmicas o centrales de biomasa de gasificación sintética, pirólisis o de combustión, requieren de mecanismos económicos adicionales, que permitan su implementación y hasta el momento el Gobierno no los ha establecido. Se puede avanzar con proyectos piloto vinculados al manejo sostenible de residuos y aprovechamiento del recurso geotermal, que no provoquen incidencia relevante en las tarifas eléctricas.

En cuanto a las limitaciones ambientales y sociales, el desarrollo de nuevos proyectos en el Perú actualmente conlleva un mayor riesgo por la obtención de permisos ambientales y licencia social en sus lugares de emplazamiento. De acuerdo con la Defensoría del Pueblo, de los 232 conflictos registrados en el 2018, el 62,1 $\%$ (144 casos) fueron de índole socioambiental (Defensoría del Pueblo, 2018). Esto denota un gran esfuerzo para los inversionistas, quienes muchas veces ven aplazados sus proyectos hasta la obtención de todos los permisos necesarios. Aunque las energías renovables gozan de mayor licencia social.

Afortunadamente, el sector eléctrico no ha tenido tantos conflictos sociales como el sector minero o petrolero; sin embargo, algunos proyectos de centrales hidroeléctricas sí los han reportado, sobre todo por la percepción que tiene la población acerca de los incumplimientos ambientales de centrales que se desarrollaron antes de la entrada en vigor de la Ley N.o 27446, Ley del Sistema Nacional de Evaluación de Impacto Ambiental (SEIA) y su Reglamento, aprobado mediante Decreto Supremo N. $.^{\circ} 019-2009-M I N A M$.

En cuanto a las limitaciones técnicas de las centrales renovables, estas varían en función de la tecnología. En el caso de las centrales hidroeléctricas de pasada, su principal limitante 
es el tiempo de construcción, que en promedio para centrales hidroeléctricas mayores a 20 MW en el Perú es de 5.6 años (Wong, 2019). Estos mayores plazos implican mayores gastos financieros durante la etapa de construcción de una central hidroeléctrica, que tendría ingresos recién al sexto año en comparación con otra tecnología. Además, estas centrales se ven limitadas por la estacionalidad del recurso e intensificación de fenómenos naturales producto del cambio climático.

En el caso de las centrales eólicas y solares fotovoltaicas, estas tienen la ventaja de contar con un menor tiempo de construcción, que en el Perú ha sido de 3 a 4 años para el caso de las eólicas y de 2.5 ańos para el caso de las solares fotovoltaicas. Esto se debe a que en realidad estas centrales no tienen etapas intensivas de construcción de infraestructura, pues los equipos se adquieren listos para la instalación. Además, los costos no tienden a subir, sino más bien a bajar de forma significativa, por las eficiencias en el desarrollo de su tecnología y economía de escala (Wong, 2019).

Las centrales eólicas y solares fotovoltaicas han demostrado ser bastante regulares a lo largo del año, alcanzando factores de planta muy competitivos a nivel mundial, siendo el promedio del factor de planta de las centrales eólicas entre $20 \%$ y $60 \%$ y el de las centrales fotovoltaicas $20 \%$. Por ello, COES ha fijado como temporal límite técnico al 2021 la participación en el sistema interconectado, de $20 \%$, de las tecnologías solar fotovoltaica y eólica. Esto es más de cuatro veces más la actual participación.

En el caso de las centrales geotérmicas, su principal limitante es el riesgo de la etapa de exploración subterránea, con un cálculo estimado promedio de costos de inversión de casi USD 4 millones por cada MW. Además, los tiempos de construc- ción de centrales geotérmicas oscilan entre 5 y 10 años en promedio, lo que retarda el retorno de la inversión (Gehringer y Loksha, 2012).

En el caso de las centrales de biomasa, las que se encuentran instaladas actualmente han tenido un tiempo de construcción promedio de 2.8 ańos (Wong, 2019), lo cual las hace atractivas; sin embargo, presentan riesgos sobre la disponibilidad del recurso de biomasa para garantizar el suministro eléctrico.

Por último, el modelo de subastas de largo plazo con RER — hasta 20 años- debe ser revisado, las tecnologías varían sus costos año a año, esto debe ser recogido en la fórmula de retribución de las licitaciones. Además, debe considerarse abrir concursos de corto y mediano plazo para atender las necesidades del mercado. Además, el mercado ha crecido y los llamados PPA (power purchase agreement) los puede organizar el sector privado, como es en Chile. Este sería un signo de crecimiento y madurez del mercado eléctrico. En el caso de los sistemas aislados, es importante la regulación de microrredes y usar diversas tecnologías renovables, además de la construcción de capacidades locales en cada pequeño centro poblado, con énfasis en el liderazgo de la mujer madre, para ganar sostenibilidad y evitar sobre costos de operación, mantenimiento y cobranza a la distribuidora de la capital de la región. Esto ayuda a la sostenibilidad de los sistemas.

\section{INTENTOS RECIENTES}

Como retos para el desarrollo de las energías renovables en el Perú, se pueden mencionar algunos criterios relevantes que han sido recogidos en gran parte por el Proyecto de Ley 6953 del Congreso de la República, presentado al Congreso Transitorio, por iniciativa de Alberto de Be- 
laúnde, en su rol de presidente de la Comisión de Cambio Climático, con el apoyo de cuatro grupos políticos. Estos aspectos relevantes son los siguientes:

- Se debe cambiar el concepto de potencia que es una barrera para las energías renovables alternativas y permitir el despacho por bloque horario. El Organismo Supervisor de la Inversión en Energía y Minería, de acuerdo con sus competencias y funciones, elabora y diseña las bases de las subastas del mercado regulado eléctrico, definiendo bloques horarios, con la finalidad de permitir la participación de nuevos proyectos de generación con RER, con el propósito de reducir el costo de la energía eléctrica domiciliaria, de acuerdo a los plazos establecidos en la Ley N. ${ }^{o}$ 28832, Ley para asegurar el desarrollo eficiente de la generación eléctrica.

- Todos los generadores deben declarar sus costos reales y debe aplicarse la libre competencia, eficiencia y transparencia en el mercado eléctrico.

- Es una tarea pendiente la implementación de una política energética de Estado que involucre el planeamiento estratégico y la participación de los diferentes actores, el Estado, la academia y la sociedad civil, con objetivos, metas en energías renovables, acciones y responsabilidades a cumplir hacia el 2030 y 2050, en línea con nuestros compromisos ambientales internacionales. En tal sentido planteo la creación de una entidad planificadora en materia energética que permita ir actualizando y monitoreando los avances en el cumplimiento de la Política Energética Nacional. Esta entidad planificadora debe tener autonomía, capacidad de decisión y recursos económicos para cumplir con las metas trazadas independientemente de los gobiernos o autoridades de turno, así como para exigir el cumplimiento de las medidas a las diferentes instituciones.

- Se debería incrementar el porcentaje de participación de las tecnologías renovables alternativas en el sistema eléctrico interconectado nacional, en base a los compromisos internacionales para hacer realidad un crecimiento sustentable con menores emisiones.

- Se debe mantener y ampliar los mecanismos de promoción para permitir el desarrollo de tecnologías renovables como la energía solar fotovoltaica, eólica, geotérmica, solar térmica y biomasa. Esto incluye la generación distribuida.

- Se debe lograr la identificación y registro digital de la cantidad total de familias a nivel nacional sin acceso a electricidad, calefacción y tecnologías limpias para cocinar, estudiando sus reales necesidades energéticas y las mejores formas de provisión de energía. Es importante que este estudio refleje la realidad social y cultural de la población y las vincule con las mejores soluciones tecnológicas a un precio accesible.

- Se debe promover el conocimiento y la innovación en el uso de energías limpias con el apoyo de la academia, generando fuentes de información accesibles a todos los ciudadanos.

- Es una tarea pendiente o truncada el incentivar la inversión en Recursos Energéticos Renovables (RER) en el mercado eléctrico peruano, con la finalidad de promover empleo, descentralización económica, reducir la brecha de acceso a la energía, promover la seguridad energética, reducir la contaminación del aire y cumplir con los com- 
promisos internacionales de reducción de emisiones de gases de efecto invernadero asumidos en el Acuerdo de París.

- El Ministerio de Energía y Minas debería establecer y ajustar cada cierto tiempo un porcentaje objetivo creciente que permita la participación la electricidad generada a partir de RER en el consumo nacional de electricidad. En el mencionado porcentaje no se debe considerar la generación de energía hidráulica. Tal porcentaje objetivo no debe ser menor a veinte por ciento $(20 \%)$ al 2030.

- Las centrales de generación de electricidad a base de RER de fuente solar y fuente eólica deberían contar con una potencia firme reconocida y remunerada equivalente a la potencia media anual inyectada en la subestación de despacho al sistema eléctrico interconectado nacional. Para las RER de fuente solar, la potencia media sería el menor valor de los últimos años de operación. Para las centrales de electricidad de fuente eólica la potencia media sería la de las horas punta del Sistema Eléctrico Interconectado Nacional (SEIN).

- Las subastas del mercado regulado deberían incluir a las energías renovables y ser convocadas atendiendo la demanda proyectada con anticipación, así como, los contratos a vencer en el mencionado plazo. Estas subastas no necesariamente son de largo plazo. Puede haber de corto y mediano plazo.

- El Ministerio de Energía y Minas debería evaluar que se promueva la instalación de plantas de producción de hidrógeno verde, utilizando como fuente energética a la electricidad generada a partir de RER como un mecanismo eficiente para reducir las emi- siones de gases de efecto invernadero de la matriz energética peruana provenientes del transporte, industria y agricultura. El Ministerio de Energía y Minas debería formular el Plan Nacional de Desarrollo del Hidrógeno Verde; establecer metas y políticas de largo plazo para fomentar la confianza de potenciales inversionistas; estimular la demanda comercial del hidrógeno, a través de múltiples usos y aplicaciones; ayudar a mitigar los riesgos, tales como la complejidad de la cadena de valor y riesgos en seguridad; promover la investigación y el desarrollo, así como el intercambio de conocimientos, y armonizar estándares y eliminar barreras.

- El Ministerio de Energía y Minas debería desarrollar un piloto de subasta para la inclusión de la generación geotérmica en el mercado, como fuente de energía constante e ininterrumpida de origen renovable que permita la descentralización de la producción de energía en el país. La inclusión de la tecnología geotérmica en la siguiente subasta RER conforme a la cuarta disposición complementaria final de la presente Ley. Esta iniciativa no debe superar los 90 MW para evitar la afectación mayor de la tarifa eléctrica en los ciudadanos.

- Se recomienda crear el canon para la explotación de los recursos energéticos renovables que se compone del $50 \%$ (cincuenta por ciento) del total de los ingresos y rentas pagado por los concesionarios que utilicen el recurso eólico, solar, geotérmico, biomasa y mareomotriz para la generación de energía, de conformidad con lo establecido por el Decreto Ley N.o 25844, Ley de Concesiones Eléctricas. 
Lamentablemente esta propuesta de Ley no fue respaldada en la Comisión de Energía y Minas del Congreso Transitorio que termina en julio del 2021. Hay otra iniciativa que tuvo mejor suerte en la Comisión de Medio Ambiente y Pueblos indígenas. Esta propuesta normativa obliga al Ministerio de Energía y Minas, en coordinación con el MINAM, a actuar bajo responsabilidad y en un plazo no mayor de 180 días, planificar e incrementar el porcentaje de participación de las energías renovables alternativas. El Ministerio de Energía y Minas, en coordinación con el Ministerio del Ambiente, debe convocar y dirigir un proceso de planificación estratégica de la transición ecológica, de carácter transversal, participativo y con un horizonte de mediano y largo plazo (2030, 2040 y 2050); con el objetivo de establecer metas de descarbonización energética, como el aumento de la participación de las energías renovables no convencionales, garantizando un sistema energético que satisfaga la demanda nacional de energía de manera regular, continua y eficiente, en la que se promueve el desarrollo sostenible. Ya conoceremos el resultado de esta propuesta legislativa, hay que estar atentos.

\section{BIBLIOGRAFÍA}

ALMONACID, G. Riesgos del sistema energético actual. En Conferencia Inaugural del Curso 1995-1996. Instituto de Estudios Giennenses. Disponible en: http://www.ujaen.es/investiga/ solar/07cursosolar/home_main_frame/08_lecciones/01_leccion/www/riesgos_del_sistema_ energetico_a.htm.

BRITANNICA ACADEMIC. Enciclopedia Británica. [en línea]. Disponible en: academic. eb.com/levels/collegiate/article/energy-conversion/106034.

BRITANNICA ACADEMIC. Enciclopedia Británica [en línea]. Disponible en: academic.eb.com/ levels/collegiate/article/energy/32627
CALAMATEO, D. y ZHOU, D. A pre-feasibility study of a small-scale concentrating solar power plant for an industrial application in $\mathrm{Sa}$ cramento, California. Proceedings of the 2nd International Conference on Fluid Flow, Heat and Mass Transfer, State University, 2015.

COMITÉ DE OPERACIÓN ECONÓMICA DEL SISTEMA INTERCONECTADO NACIONAL (COES SINAC).. Informe mensual de operación. Lima: Comité de Operación Económica del Sistema, 2020a.

COMITÉ DE OPERACIÓN ECONÓMICA DEL SISTEMA INTERCONECTADO NACIONAL (COES SINAC). Informe de la operación anual del SEIN 2019. Lima, COES, 2020 b.

CONGRESO DE LA REPÚBLICA. Decreto Supremo N. ${ }^{\circ}$ 064-2010-EM [Política Energética Nacional del Perú, 2010-2040]. Lima: Diario El Peruano, 2010.

CONGRESO DE LA REPÚBLICA. Decreto Legislativo N. ${ }^{\circ} 1002$, Decreto Legislativo de promoción de la inversión para la generación de electricidad con el uso de energías renovables, Lima: Diario El Peruano, 2008.

CRUZ, V. y VARGAS, V. Geothermal country update for Peru, 2015-2020. En: Proceedings World Geothermal Congress 2020, Reykjavik, Iceland, 2019.

DAMMERT, A. Las reformas en el mercado de generación eléctrica en el Perú. [Entrada en blog]. Blog de la Maestría en Regulación de los Servicios Públicos. Lima, 1 de febrero 2018. Recuperado de shorturl.at/swO68

DEFENSORÍA DEL PUEBLO. Vigésimo Segundo Informe Anual 2018. Lima: Defensoría del Pueblo, 2018.

DEUTSCHE GESELLSCHAFT FÜR INTERNATIONALE ZUSAMMENARBEIT (GIZ. Waste-to-energy options in municipal solid waste management.) GmbH, Bonn and Eschborn, Germany, 2017.

DIPIPPO, R. Geothermal power plants. principles, applications, case studies and environmental impact. 4. ${ }^{a}$ ed. Butterworth-Heinemann, 2015.

ELLIOT, P. Visión politica y estado actual de las energías renovables en el Perú. Lima, 2019.

EMISSIONS, G. Comparison of lifecycle greenhouse gas emissions of various electricity generation sources. London: 2011. 
FEDERAL MINISTRY FOR THE ENVIRONMENT, NATURE CONSERVATION AND NUCLEAR SAFETY. Sustainable energy production from biomass waste in Peru. NAMA Proposal. German Bundestag, 2015.

FRIDRIKSSON, T.; MATEOS, A.; AUDINET, P.; ORUCU, Y. Greenhouse gases from geothermal power production. Energy Sector Management Assistance Program (ESMAP) Technical Report 09/16. Washington, D. C.: World Bank, 2016. Disponible en: https://openknowledge. worldbank.org/handle/10986/24691

GEHRINGER, M. y LOKSHA, V. Manual de geotermia: Cómo planificar y financiar la generación de electricidad. ESMAP Technical Report 002/12. World Bank, Washington, D. C. 2012. Disponible en: https://openknowledge.worldbank. org/handle/10986/23712

GOBIERNO DEL PERÚ. Exposición de motivos de la Política Energética Nacional 2010- 2040, Lima: Diario El Peruano, 2012.

GOBIERNO DEL PERÚ. Informe final del grupo de trabajo multisectorial de naturaleza temporal encargado de generar información técnica para orientar la implementación de las contribuciones nacionalmente determinadas (GTM-NDC). Lima: Gobierno del Perú, 2018.

GOBIERNO DEL PERÚ. Plataforma digital única del Estado peruano [en línea]. Disponible en: https://www.gob.pe/.

GREEN ENERGY. Estudio para la promoción de la generación eléctrica con fuentes de energía renovable. Lima: Ministerio de Energía y Minas, Dirección General de Electricidad, 2005.

INTERGOVERNMENTAL PANEL ON CLIMATE CHANGE. Renewable Energy Sources and Climate Change Mitigation. Summary for Policymakers and Technical Summary. New York: Cambridge University Press, 2011.

INTERNATIONAL BANK FOR RECONSTRUCTION AND DEVELOPMENT. State of electricity access report 2017. Washington D.C.: The World Bank, 2017, 110 pp.

INTERNATIONAL ENERGY AGENCY. Global CO2 emissions in 2019. International Energy Agency, 2020.

INTERNATIONAL FINANCE CORPORATION. Success of geothermal wells: A global study. World Bank Group, 2013.
INTERNATIONAL FINANCE CORPORATION. Converting biomass to energy. A guide for developers and investors. Washington D.C., 2017. Disponible en: http://hdl.handle. net/10986/28305

INTERNATIONAL RENEWABLE ENERGY AGENCY. Global land outlook working paper. IRENA, 2017.

INSTITUTO NACIONAL DE ESTADÍSTICA E INFORMÁTICA (INEI). Censos Nacionales 2017: XII de Población, VII de Vivienda y III de Comunidades Indígenas. Lima, 2017. Disponible en: http://censos2017.inei.gob.pe/ redatam/

JAGANMOHAN, M. Global LCOE of hydropower energy 2010-2019. Disponible en: https://www. statista.com/statistics/799349/lcoe-of-hydropower-worldwide/

LAZARD. Lazard's levelized cost of energy analysis v13.0. 2019.

MATEK, B. Geothermal energy association issue brief: firm and flexible power services available from geothermal facilities. Washington D. C.: Geothermal Energy Association, 2015.

MINISTERIO DE ENERGÍA Y MINAS (MINEM). Plan Energético Nacional 2014-2025. Lima: Ministerio de Energía y Minas, 2014.

MINISTERIO DE ENERGÍA Y MINAS (MINEM). Perú, subsector eléctrico. Documento promotor 2012. Lima: Ministerio de Energía y Minas, 2012.

MINISTERIO DE ENERGÍA Y MINAS (MINEM). Portal del Ministerio de Energía y Minas. Disponible en: http://www.minem.gob.pe/.

MINISTERIO DE ENERGÍA Y MINAS (MINEM). Atlas eólico del Perú. Lima: Ministerio de Energía y Minas, 2016.

MINISTERIO DE ENERGÍA Y MINAS (MINEM). Atlas del potencial hidroeléctrico del Perú. Lima, Ministerio de Energía y Minas, 2011.

MINISTERIO DEL AMBIENTE. Sistema Nacional de Información Ambiental 2020. [En línea]. Disponible en: https://sinia.minam.gob.pe/ indicador/ 1600

NACIONES UNIDAS. Objetivo 7: Garantizar el acceso a una energía asequible, segura, sostenible y moderna. Disponible en: https://www. un.org/sustainabledevelopment/es/energy/ 
ORGANISMO SUPERVISOR DE LA INVERSIÓN EN ENERGÍA Y MINERÍA (Osinergmin). La industria de la electricidad en el Perú: 25 años de aportes al crecimiento económico del país. Lima: Organismo Supervisor de la Inversión en Energía y Minería, 2016, pp. 176.

PANEL INTERGUBERNAMENTAL SOBRE EL CAMBIO CLIMÁTICO (IPCC). Informe especial. Calentamiento Global de $1.5^{\circ} \mathrm{C}$. IPCC, 2018.

PLANAS, O. «Energía solar». [Consulta en línea, 29 mayo 2020]. Recuperado de https://solar-energia.net/energia-solar-termica/central-solar-termica.

¿Qué es la energía eólica y cómo funciona? Recuperado de: https://www.enel.pe/es/sostenibilidad/quees-la-energia-eolica-y-como-funciona.html

¿Qué es la energía solar fotovoltaica y cómo funciona? Recuperado de: shorturl.at/inKRS

REN 21. Renewables 2020 Global Status Report. 2020.

ROLDÁN, M. Concentrating solar thermal technologies: Analysis and optimisation by CFD modelling. Springer, 2017.

SECRETARÍA DE ENERGÍA-REPÚBLICA ARGENTINA. Centrales eléctricas. 2012. Recuperado de: shorturl.at/qEY37
SERVICIO NACIONAL DE CERTIFICACIÓN AMBIENTAL PARA LAS INVERSIONES SOSTENIBLES (SENACE). Manual de evaluación del estudio de impacto ambiental detallado (EIA-d) para el subsector electricidad. Lima: Servicio Nacional de Certificación Ambiental para las Inversiones Sostenibles, 2016.

SOVACOOL, B. K.; NUGENT, D. y Gilbert, A. "Construction cost overruns and electricity infrastructure: An unavoidable risk? The Electricity Journal, vol. 27, n. ${ }^{\circ}$ 4, pp. 112-120, 2014.

THE WORLD BANK, ESMAP Y SOLARGIS. Solar resource maps of Peru. The World Bank, 2019 [en línea]. Recuperado de: https://solargis. com/maps-and-gis-data/download/peru.

VARGAS, V. y CRUZ, V. Geothermal map of Peru. Lima, Instituto Geológico Minero y Metalúrgico, 2010.

VÁSQUEZ, A.; TAMAYO, J. y JULIO SALVADOR (editores). La industria de la energía renovable en el Perú: 10 años de contribuciones a la mitigación del cambio climático. Lima-Perú: Osinerg$\min , 2017$.

WONG, L. Evaluación de tecnologías para suplir la demanda de generación eléctrica en el Perú al 2022. Lima: Universidad de Ingeniería y Tecnología, 2019. Recuperado de: https://repositorio.utec. edu.pe/handle/20.500.12815/123 